CHAPTER 6

\title{
Word Skipping: Implications for Theories of Eye Movement \\ Control in Reading
}

Marc Brysbaert

University of Ghent

and

Francoise Vitu

Universite Rene Descartes

\begin{abstract}
Eye movements in reading are characterized by short periods of steadiness (fixations) followed by fast movements (saccades). Saccades are needed to bring new inform-ation into the centre of the visual field where acuity is best; fixations are required to recognize words. Assuming that the central (foveal) word is identified during a fixation, it is tempting to forward the hypothesis that eye movements in reading essentially consist of word-to-word movements. Unfortunately, such a simple sequence of motion is rarely observed in empirical data. Some words are fixated more than once, some are initially not fixated but immediately afterwards regressed to, and some are not fixated at all. Ever since the first measurements of eye movements in reading, researchers have been puzzled by this complicated pattern of activity and have suggested various explanations for it. In this chapter, we will focus on one aspect, namely the fact that more than one third of the words are initially skipped during reading. First, we will discuss the explanations offered by different authors, then we will examine the empirical evidence more closely, and, finally, we will present an alternative account of word skipping.
\end{abstract}




\section{Word skipping in different theories}

The loose relation between eye movements and text layout made many of the first researchers believe that eye movements were controlled by an autonomous oculo-motor control centre (e.g., Buswell, 1920; Erdman and Dodge, 1898; Huey, 1908). According to this view, saccade sizes were more or less constant and only changed as a function of the global difficulty of the materials being read. Variations in saccade size resulted from noise in the oculomotor system and adjustments to the difficulty of the text. As for word skipping, this implied that the probability of skipping a word depended on the overall easiness of the text but not on the easiness of the word itself.

The autonomous oculomotor control model remained the dominant model until the middle of the 1970s, although some refinements were added. For instance, Bouma and de Voogd (1974; see also Shebilske, 1975) attributed changes in saccade size to the limited capacities of an input buffer which could contain but a few activated word units. According to this view, it is only when the buffer gets full or empty that saccade sizes vary around their means. Other variants of the model speculated that variations in saccade size were determined by visual characteristics of the words, in particular the length of the upcoming, parafoveal, word. For example, having found that the length of inter-word saccades depended on the length of the parafoveal word, O'Regan (1980) hypothesized that the eyes were programmed to jump to the centre of the next word. Again, word skipping was not assumed to depend on the easiness of the word (but see O'Regan, 1979).

Gradually, the possibility of linguistic influences on the probability of word skipping began to be taken into account. In his cognitive and peripheral search guidance theory, Hochberg $(1975,1976)$ still maintained that eye movements were primarily determined by pre-established scanning routines independent of the ling-uistic information extracted at each fixation, but these routines depended on both the readers' knowledge of the language constraints and on the task they set to them-selves when reading. If the task required subjects to pay attention to the letters and the spelling of the words without paying attention to the meaning (proofreading), readers were assumed to adopt a letter-by-letter or word-by-word scanning routine. If, however, the reading purpose was to extract meaning from text, then larger saccades were made in order to reduce the number of samples per line. In that case, the size of the saccades was controlled by peripheral search guidance mechanisms: At each fixation, the reader anticipated what he would find next on the basis of the meaning and the grammar status of the words previously read and on the basis of global visual information extracted in parafoveal vision (such as the next word's length). Readers directed their eyes to the portions of text which appeared as the most informative to test the predictions that had been made. So, according to Hochberg, the size of a saccade depended on (i) text difficulty and redundancy 
(which affect a word's predictability and the size of the perceptual span), and (ii) the reader's ability to extrapolate upcoming information and to process parafoveal stimuli.

Similar reasoning can be found in Shebilske (1975). Saccade sizes were mainly determined by the capacities of the input buffer (see above), but on some instances the ongoing linguistic processing could intervene. If both the overall meaning of the words read on previous fixations and the visual information extracted from the parafovea (such as the next word's length) made the next word highly predictable, then the automatic oculomotor program could be interrupted and the word skipped. This could occur independently of the state of the internal buffer. So, linguistically induced word skipping was possible when the word was highly predictable from prior context and the parafoveal visual information compatible with this prediction.

In the late 1970s, the idea that word skipping did not happen at random but was determined by the probability of the parafoveal word being identified during the previous fixation, was strongly promoted in a series of highly influential papers. McConkie (1979) hypothesized that saccade length was determined by the size of the perceptual span (see also Rayner, 1978). Saccades were tuned at each fixation in order to bring the eyes to the region of text which was not clearly visible from the current fixation location. Therefore, saccade sizes were determined by visual acuity limitations, but also by the ease of processing associated with the words to the right of the fixation location (i.e., their predictability and their frequency).

Further support for the processing account of word skipping was provided by the different variants of the attentional theory of eye guidance in reading (Morrison, 1984; Pollatsek and Rayner, 1990; Henderson and Ferreira, 1990; see also Chapter 1 I for the most recent developments). According to this theory, during reading there is an interplay between attentional processes and eye movement control. As orig-inally proposed by Morrison (1984; see also McConkie, 1979), the sequence of events within a fixation is as follows. Initially, attention is focused on the word in foveal vision. As soon as this word is identified, the attentional beam shifts toward the next word and this word starts to be processed. The shift of attention triggers the programming of a saccade, which is executed after a more or less fixed delay. If processing of the parafoveal word is completed some time before the end of the delay, word skipping is possible. Because the parafoveal word has been identified, the attentional beam again shifts to the next word, and the saccade to the first parafoveal word will be cancelled and replaced by a saccade to the second word. As the probability of parafoveal word identification before saccade execution depends on the easiness of the parafoveal word, the attentional theory predicts that all word skipping should be linguistically based.

Although the attentional theory has dominated eye movement research during the last decade, the idea that word skipping may be controlled by non-linguistic 
strategies has not completely vanished. For instance, Just and Carpenter $(1980,1987)$ argued that saccade sizes were independent of on-going processing require-ments, and were preprogrammed so that the eyes fixated every word and landed between the beginning and the middle of a word. The hypothesis was that the information extracted from the parafovea was not very detailed, and served mainly to locate the target word for the next saccade. This information was assumed not to he clear enough for recognition of the parafoveal word to take place and to influence the length of the next saccade. Only in exceptional cases when the eyes were located at the very end of a word and when the next word was very short and frequent was it possible that parafoveal word recognition occurred and led to word skipping. So, according to Just and Carpenter, word skipping was largely independent of the easiness of the parafoveal word, and the variability in saccade sizes mainly resulted from visual and oculomotor factors. Just and Carpenter's view on landing sites is particularly surprising, given how high-level the rest of their theory on eye move-ment control in reading was.

A very similar argument can be found in O'Regan's (1990) strategy-tactics theory. According to this theory, the inter-word behaviour of the eyes was mainly determined by a preset oculomotor strategy that depended on the global difficulty of the text and on task demands. O'Regan basically made a distinction between a careful reading strategy (i.e., going from word to word) and risky reading strategics (e.g., skipping every second word, only looking at long words). A further distinction was made between the saccade target (i.e., the middle or the optimal viewing position of a parafoveal word) and the actual landing position. According to O'Regan, visuomotor constraints considerably reduce the accuracy with which a saccade can be executed during reading. So, in the strategy-tactics theory, there were two main determinants of word skipping. Words were skipped because of the strategy (e.g., because they were too short) or because of an oculomotor error (the eyes jumped too far). In addition, O'Regan (1990) accepted linguistic influences if the previous fixation exceeded a certain duration. In this case, parafoveal word processing could interfere with the oculomotor strategy.

Finally, it should be noted that McConkie in more recent publications (e.g., McConkie, Kerr and Dyre, 1994) returned to the idea of independent oculomotor strategies during reading. Having found that the probability of word skipping can he predicted rather well by equations that only involve word length and launch site (see below), he started to question the viability of linguistic control theories. To put it in his own words: "...we have briefly outlined the approach we are taking in our attempt to produce a mathematical model of the eye movements of normal, skilled readers. Our greatest surprise thus far has been to observe how much of the variance in the data can be accounted for with a relatively few parameters, and these often reflecting such low-level variables as word length, eye position in word and launch site." (McConkie et al., 1994, p. 325). 


\section{Empirical data}

In this section we will look at the empirical evidence upon which the above theories are based. We will deal successively with the landing position distribution, the effects of word length and launch site, and the contribution of language factors such as word frequency and the extent to which a word is constrained by the sentence context. Finally, we will try to determine the relative importance of visual and linguistic variables, in order to decide which should come first in an acceptable model.

\section{Landing position distribution}

Although the impact of oculomotor errors on eye movements in reading has been defended most strongly by O'Regan and colleagues (e.g., Coeffe and O'Regan, 1987; O'Regan, 1990), it was McConkie and his group who presented the most convincing empirical evidence for such an effect (McConkie et al., 1988, 1994; see also Radach and Kempe, 1993). A typical study involved a few participants reading a complete novel, so that analyses could be based on a large number of observations per person. One of the major analyses was the frequency distribution of initial fixations on a word as a function of the launch site (i.e., the letter position relative to the word centre from which the eye movement started) and the length of the target word. Figure 1 depicts the prototypical findings. There are two main effects. First, the distribution of landing positions is well captured by a Gaussian curve, and

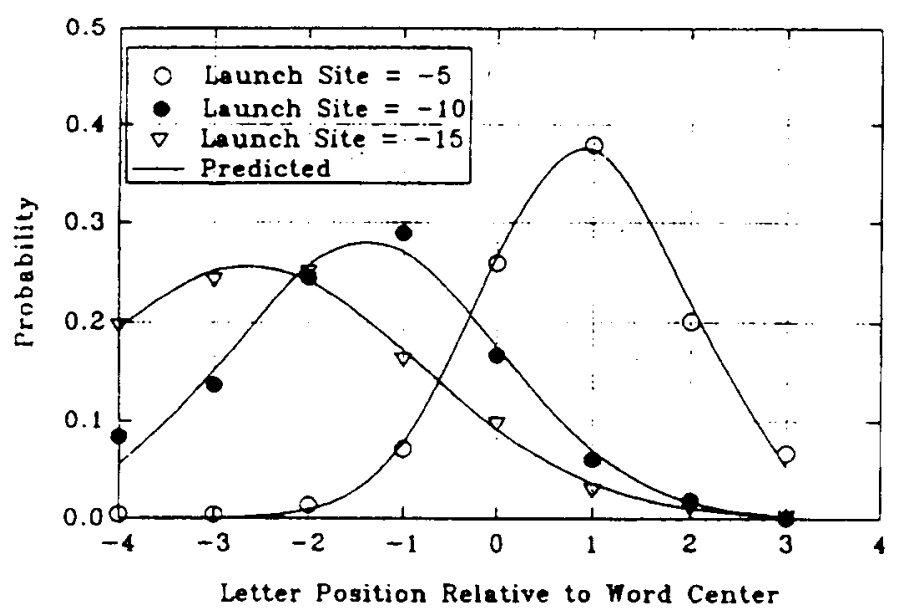

Fig. I. Frequency distributions of initial fixations on 7-letter words, following saccades launched

from 5, 10, and 15 letter positions to the left of the center of the word (reprinted from MeConkie et al., 1994). 
second, the mean of the distribution is a function of the launch site. For each launch site one character position farther to the left, the mean of the landing position distribution moves leftward by about a third of a character position. McConkie attributes this to a range error, by which the system tends to overshoot near targets and undershoot far targets.

So far, the landing position distribution has mainly been used by McConkic and colleagues to explain the distribution of initial fixations within words. However, as can be seen in Fig. I, the pattern can easily be extended to the between-words case (i.e., word skipping), as has been asserted by O'Regan (see above). The strategy-tactics theory further draws attention to two other visuomotor constraints. The first refers to the fact that the landing error is likely to be larger after short fixation durations than after long fixation durations (Coeffe and O'Regan, 1987). The second points to the fact that eye movements to a target are influenced by the presence of other targets in the visual field (i.e., the global effect; Findlay, 1982; Vitu, 1991 a). Given that words of a text are not presented in isolation, the claim is that the properties of the words behind the target word will influence the saccade size towards the target word. This is particularly true for short target words.

On the basis of the present evidence, it is beyond doubt that all comprehensive theories of word skipping should take the existence of involuntary word skipping due to oculomotor error into account (as well as the possibility that a word is involuntarily looked at due to a saccade undershoot). What is less sure, however, is whether all deviations from O'Regan's deterministic inter-word strategy can be explained by oculomotor errors. This question is especially relevant when one looks at the effects of word length and launch site on word skipping.

Word length an(1 launch site

One of the most conspicuous aspects of word skipping is that it happens more often with short words than with long words. For instance, Vitu et al. (1995) reported skipping probabilities of about $80 \%$ for one-letter words, $60 \%$ for three-letter words, $30 \%$ o for five-letter words, and $10 \%$ for words of seven letters or longer. Interestingly, these figures were virtually the same whether words in real sentences were presented, or meaningless z-strings that had the same layout (cf., the issue of mindless reading; Vitu et al., 1995; Rayner and Fischer, 1996).

Kerr (1992) was the first to note that the average word length effect hides another, equally strong, effect of launch site. Figure 2 shows some typical data. They are drawn from a study in which 24 participants read 120 sentences (see Brysbaert and Mitchell, 1996, Experiment 3 for further details), resulting in a total of more than 22,000 observations for words from two letters to nine letters. Skipping rate is plotted as a function of word length (2-9 letters) and launch site (1-15 letters; operationalized as the distance in letter positions from the blank space in front of the 


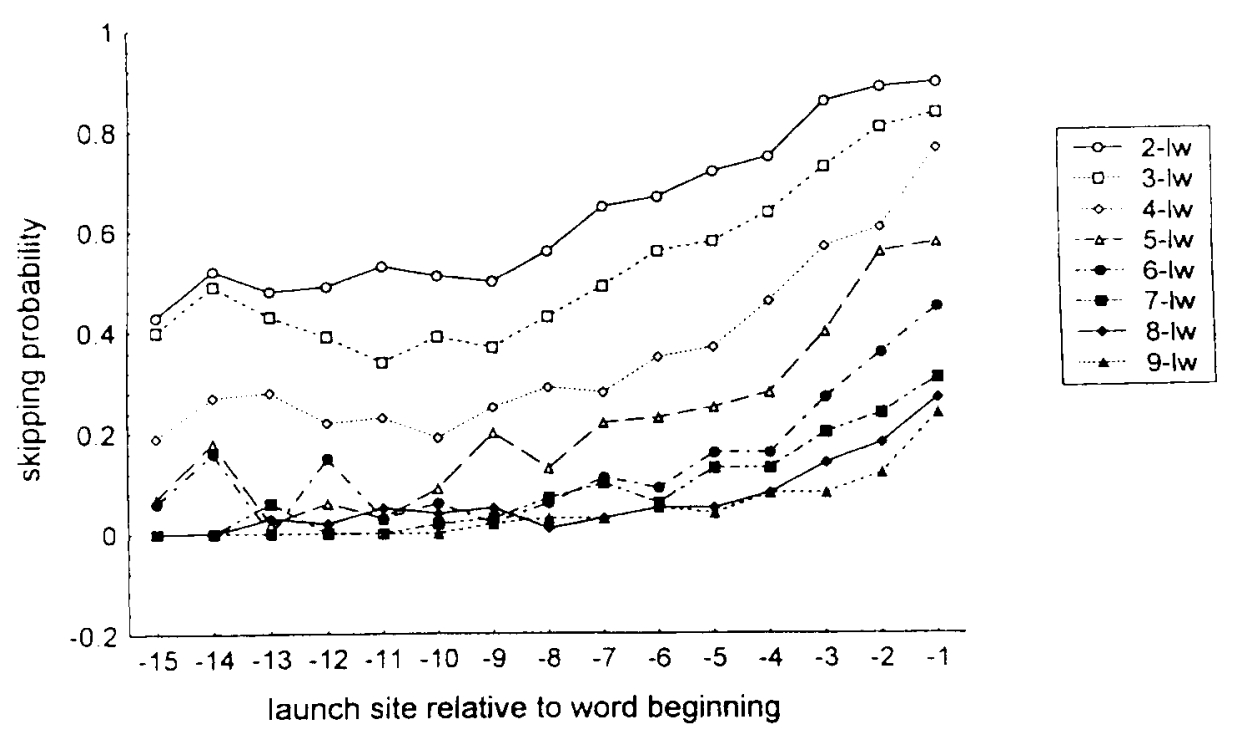

Fig. 2. Word skipping probability as a function of word length and launch site in letter positions

relative to the blank space in front of the word.

word). Although a two-letter word on the average was skipped on $69 \%$ of the cases, this figure ranged from $90 \%$ at launch site one (the last letter of the previous word) to some $50 \%$ at launch site 15 (when the eyes already had skipped one or two words). Similar data have been reported by Kerr (1992), Vitu et al. (1995), and Rayner, Sereno, and Raney (1996). In addition, Vitu et al. (1995) showed that the effect of launch site on skipping rate also applies for meaningless z-strings.

It should be noted that the word length and the launch site effect can equally well be explained by theories that are based on autonomous oculornotor scanning strategies and theories that are based on linguistic control. According to the oculo-motor control theories, short words and words close to the launch site are skipped more often because they lie in an area of high visibility (cf. acuity drops away from the fixation location) and/or because they are less easy to land upon (e.g., due to the landing position error and the global effect). According to the linguistic control theories, short words and words close to the launch site are skipped more often because they are more likely to be identified before saccade onset. This is again due to the drop of visibility outside the fixation position and, in the case of word length, to the fact that short words in general are easier than long words (e.g., they usually have a higher frequency). In order to decide between both types of theories, researchers have tried to disentangle length and processing difficulty by looking at skipping rates for carefully controlled stimulus words and sentences. 


\section{Language factors}

The only way to find out whether parafoveal words are skipped because they were identified during the previous fixation, is to examine skipping rate for stimulus materials that are identical except for the target word. Otherwise, it is impossible to disentangle the effects of the scanning strategy (which can be a function of the difficulty of the text up to the target word and of the length of the words in front of the target word) from those of language processing. In addition, a difference may he drawn between studies in which the processing load of the target word was a function of properties of the word itself, and studies where the processing load was a function of the extent to which the word was constrained by the previous words in the sentence. As not all models predict an equal effect size for both cases (e.g., Hochberg and Shebilske predict a larger effect of contextual constraints than of lexical properties), we will discuss them separately.

We were able to locate seven studies in which skipping rates were compared for easy and difficult parafoveal words (see Table 1). In five of these studies, the variable that was manipulated, was the frequency of the word. For instance, Rayner and Fischer (1996) compared sentences like "He invested his money to build a store and was soon bankrupt." with sentences like "He invested his money to build a wharf and was soon bankrupt". In the other two studies, the manipulation was whether the word was visible in parafoveal vision or not up to the moment the eyes crossed the blank space in front of the word. As can be seen, in all studies and for all word lengths, skipping rate was higher or equal in the easy condition than in the difficult condition. This establishes beyond doubt that lexical variables do influence the probability of word skipping. On the other hand, it should be noted that the overall difference in skipping rate is rather small $(4 \%)$ and tends to be slightly larger for short words than for long words.

Eight other studies looked at the effects of contextual constraints on word skipping (see Table 2). Contextual constraints are usually measured by examining how many participants fill in a particular word in a doze task. For instance, given the sentence "The woman took the warm cake out of the , participants are much more likely to fill in "oven" (93\%) than "pantry" (3\%) (Schwanentlugel, 1986). Again, in all studies we found, the difference was in the expected direction: predicted words were skipped more often than unpredicted words. The mean difference amounted to $9 \%$. The largest effect (of $23 \%$ ) was reported by Vonk (1984) who (in Dutch) compared sentences like "Mary was envious of Helen because she never looked so good", where the pronoun had no disambiguating value, with sentences like "Mary was envious of Albert because she never looked so good", where the pronoun did disambiguate (in an unexpected continuation of the sentence). Brysbaert and Vitu (1995) used the same materials, but compared sentences with an expected continuation "Mary was envious of Marc because he 
Table 1

Skipping rate as a function of word characteristics

\begin{tabular}{|c|c|c|c|c|c|}
\hline Study & Manipulation & $\begin{array}{l}\text { Word length } \\
\text { (letters) }\end{array}$ & $S_{\text {rasy }}$ & $S_{\text {titit }}$ & Diff. \\
\hline \multirow{3}{*}{$\begin{array}{l}\text { Blanchard et al. (1989) } \\
(\exp 1+2)\end{array}$} & \multirow[t]{3}{*}{ parafov preview } & $1-3$ & 0.56 & 0.43 & 0.13 \\
\hline & & $4-5$ & 0.20 & 0.10 & 0.10 \\
\hline & & $6-10$ & 0.06 & 0.04 & 0.02 \\
\hline Pollatsek et al. (1992) & parafov preview & $3-8$ & 0.12 & 0.10 & 0.02 \\
\hline $\begin{array}{l}\text { Henderson and Ferreira } \\
\text { (1993) }\end{array}$ & word frequency & $4-7$ & 0.18 & 0.18 & 0.01 \\
\hline $\begin{array}{l}\text { Inhoff and Topolski (1994) } \\
(\exp 2+3)\end{array}$ & word frequency & $4-7$ & 0.27 & 0.18 & 0.09 \\
\hline \multirow{6}{*}{ Rayner et al. (1996) } & \multirow[t]{6}{*}{ word frequency } & 5 & 0.20 & 0.14 & 0.06 \\
\hline & & 6 & 0.19 & 0.16 & 0.03 \\
\hline & & 7 & 0.13 & 0.12 & 0.01 \\
\hline & & 8 & 0.09 & 0.08 & 0.01 \\
\hline & & 9 & 0.06 & 0.06 & 0.00 \\
\hline & & 10 & 0.08 & 0.07 & 0.01 \\
\hline Rayner and Raney (1996) & word frequency & $6-9$ & 0.17 & 0.11 & 0.06 \\
\hline \multirow[t]{6}{*}{ Rayner and Fischer (1996) } & \multirow[t]{6}{*}{ word frequency } & 5 & 0.18 & 0.08 & 0.10 \\
\hline & & 6 & 0.12 & 0.05 & 0.07 \\
\hline & & 7 & 0.05 & 0.01 & 0.04 \\
\hline & & 8 & 0.02 & 0.02 & 0.00 \\
\hline & & 9 & 0.03 & 0.02 & 0.01 \\
\hline & & & & & 0.04 \\
\hline
\end{tabular}

always looked so good" and sentences with an unexpected continuation "Mary was envious of Marc because she never looked so good". They found a difference in skipping rate of $9 \%$.

Rayner and Well (1996) pointed out that the difference in contextual constraints between predicted and unpredicted words has to be rather large in order to obtain an effect on skipping rate. For example, in their first experiment, Ehrlich and Rayner (1981) had a difference between $93 \%$ and $15 \%$ continuations in the doze task and 
Table 2

Skipping rate as a function of contextual predictability

\begin{tabular}{|c|c|c|c|c|c|}
\hline Study & Manipulation & $\begin{array}{l}\text { Word length } \\
\text { (letters) }\end{array}$ & $S_{\text {taty }}$ & $S_{\text {tlit1 }}$ & Diff. \\
\hline $\begin{array}{l}\text { Ehrlich and Rayner (1981) } \\
(\exp 1+2)\end{array}$ & context. constr. & 5 & 0.41 & 0.35 & 0.06 \\
\hline Balota et al. (1985) & context constr. & $4-8.0 .11$ & 0.02 & 0.09 & \\
\hline Vonk (1984) & pronoun pred. & 3 & 0.40 & 0.17 & 0.23 \\
\hline Schustack et al. (1987) & context. constr. & $3-8$ & 0.28 & 0.16 & 0.12 \\
\hline Hyona (1993) & context. constr. & $7-10$ & 0.04 & 0.00 & 0.04 \\
\hline $\begin{array}{l}\text { Inhoft and Topolski (1994) } \\
(\exp 1)\end{array}$ & word consistency & $4-7$ & 0.13 & 0.07 & 0.06 \\
\hline Brysbaert and Vitu (1995) & pronoun pred. & 3 & 0.49 & 0.40 & 0.09 \\
\hline \multirow[t]{2}{*}{ Rayner and Well (1996) } & context constr. & $4-8$ & 0.22 & 0.10 & 0.12 \\
\hline & & & & & 0.09 \\
\hline
\end{tabular}

reported skipping rates of $49 \%$ vs. $38 \%$. In their second experiment, however, continuations were only $60 \%$ and $0 \%$, and no difference in skipping rate was found (32\%o in both cases). Similarly, Hyona (1993) compared conditions of $65 \%$ and $321 /$ continuation and found virtually no effect on word skipping ( $4 \%$ c vs. $0^{\prime} / O$. To test the effect of context constraints directly, Rayner and Well (1996) compared three conditions: one in which the target word had been given by $86 \%$ of the raters in a cloze task, one in which the target word had been given by $41 \mathrm{C} /$ of the raters, and one in which the target word had been given by only $4 \%$ of the raters. Skipping rates were respectively $22 \%, 12 \%$, and $10 \%$; that is, virtually no difference was found between the 41 $\%$ and the $4 \%$ condition.

The relative importance of visual and linguistic variables

Thus far, we have shown evidence for oculomotor, visual, and linguistic influences on word skipping. However, it should be noted that much of this evidence is not fully conclusive. For instance, the data of the landing position distributions (Fig. I ) have been obtained by aggregating over different word frequencies (and other lexical variables) and different text fragments before the eyes reached the launch site. Similarly, the effects of word length and launch site (Fig. 2) are usually 
reported without reference to the frequency of the words in the different cells. An exception to the latter can be found in Rayner et al. (1996) (Fig. 2) who looked at the effects of launch site and word frequency on the skipping rate for five- and six-letter words. They reported independent effects of word length and launch site, together with a frequency effect at close launch sites (up to three letter positions in front of the target word). However, even in this study, the text fragments on which the data were based may have been quite different in the various conditions (e.g., it is not unlikely to assume that certain sequences of word lengths resulted in far launch sites and others in near launch sites, or that high-frequency and low-frequency words appeared in different sequences of word lengths).

This unsatisfactory state of affairs has motivated theorists of eye movement control in reading to stay on their opposite positions. Much of the debate has centred around the presence or absence of statistically significant differences in skipping rate between easy and difficult conditions, and on imposing further constraints on what are good stimulus materials and empirical data (e.g., according to O'Regan, one should exclude cases of long fixation durations because in these exceptional instances the ongoing processing may interfere with the autonomous scanning strategy; unfortunately, O'Regan has never specified what are "Ion-" fixation cl mations).

However, there may be a way out of the debate by capitalizing more on the data of Tables I and 2. In these tables, we have skipping rates for words that were presented in identical sentences and which were constructed in such a way as to maximize the difference in processing difficulty (e.g., by having a large difference in frequency or in contextual constraint). So for these stimuli, word length should not have an effect on skipping rate if eye movements are entirely under the control of the ongoing processing. That is, for these studies we must not find that difficult threeletter words are skipped more often than easy five-letter words. The relative contribution of word length and processing difficulty can be determined by running a multiple regression analysis with both variables as predictors and skipping rate as dependent variable. This is the more easy because the predictors in Tables I and 2 are orthogonal.

Although we could have taken the raw values of word length, we opted for an exponential transformation, which has the advantages that (1) larger weights are given to differences between short words than between large words, and (ii) that the function asymptotes to I (for word length zero) and to 0 (for very long words). Ideally, processing ease of the words should also be part of the exponential function (in order to retain the advantage of the asymptotic values), but for reasons of clarity we introduced this factor as a separate variable in the equation. So, skipping rate is predicted as a function of $\exp$ (word length) and easiness of the word (operation-alized as -0.5 for the difficult condition and +0.5 for the easy condition). There are 36 data cells in Table I, and 16 in Table 2. 


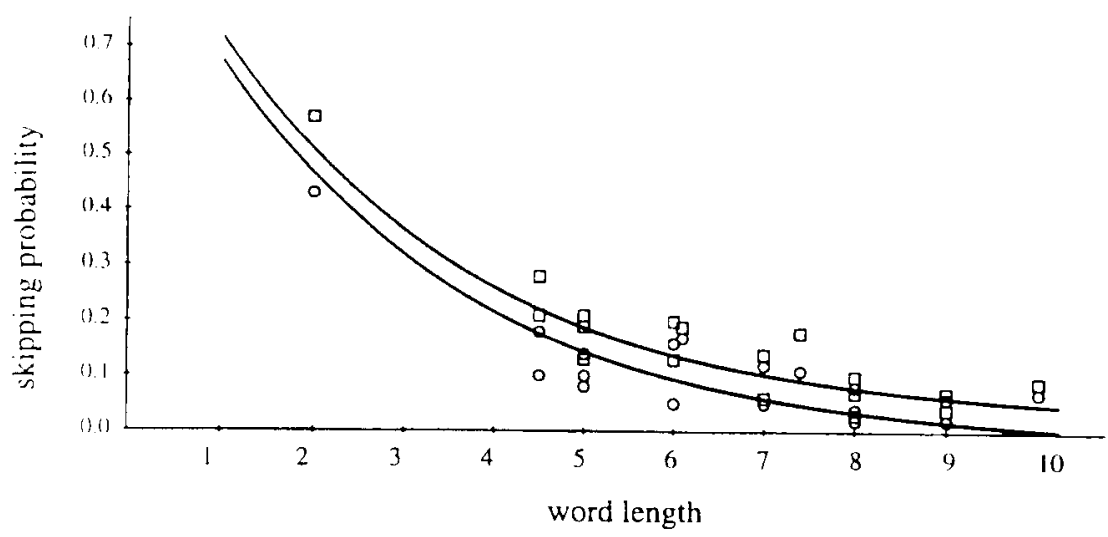

Fig. 3. Skipping rate as a function of word length and word difficulty (circle = easy condition;

square $=$ difficult condition). Empirical data from Table 1. Fitted curve based on nonlinear regression with $\operatorname{exp(word~length)~and~contextual~constraint~as~predictors.~}$

Figure 3 shows the outcome for the data of Table I (processing load due to word characteristics). Parameters were estimated by means of nonlinear regression anal-ysis. The resulting equation is:

/cskipping $=100\left(\mathrm{e}^{-\mathrm{A}} \cdot ;\right.$ ' " od $\mathrm{d}^{\text {length })}+4.3$ processing load

Both regression weights were significant (word length: $\mathrm{t}(34)=-29.4, \mathrm{p}<0.01$; processing load: $\mathrm{t}(34)=2.73, \mathrm{p}<0.01$ ) and together accounted for $83 \%$ of variance, of which $79 \%$ was due to word length and $4 \%$ to processing load.

Figure 4 shows the results for Table 2 (processing load due to context const-raints). A problem here was that many studies did not give enough details to calculate the exact mean word length. In these cases, the average of the lengths reported was used as predictor (a better but more complex approach would have been to take the geometric mean). The resulting equation is:

${ }^{1} 1 \mathrm{c}$ skipping $=100\left(\mathrm{e}^{-1, \mu^{\prime \prime},{ }^{\circ} \mathrm{d} \text { vn th }}\right)+10.0$ processing load

The regression weight of word length was highly significant, the one of processing load reached significance only for a one-tailed test (word length: $\mathrm{t}(15)=-11.6, \mathrm{p}<0.01$; processing load: $\mathrm{t}(15)=1.86, \mathrm{p}<0.10$, two-tailed). The regression accounted for $55 \%$ of variance, of which $44 \%$ was due to word length and I I \% to processing load.

The main conclusion from Figs. 3 and 4 is that even for studies which specifically looked at the effects of processing difficulty on skipping rate, word length was a 


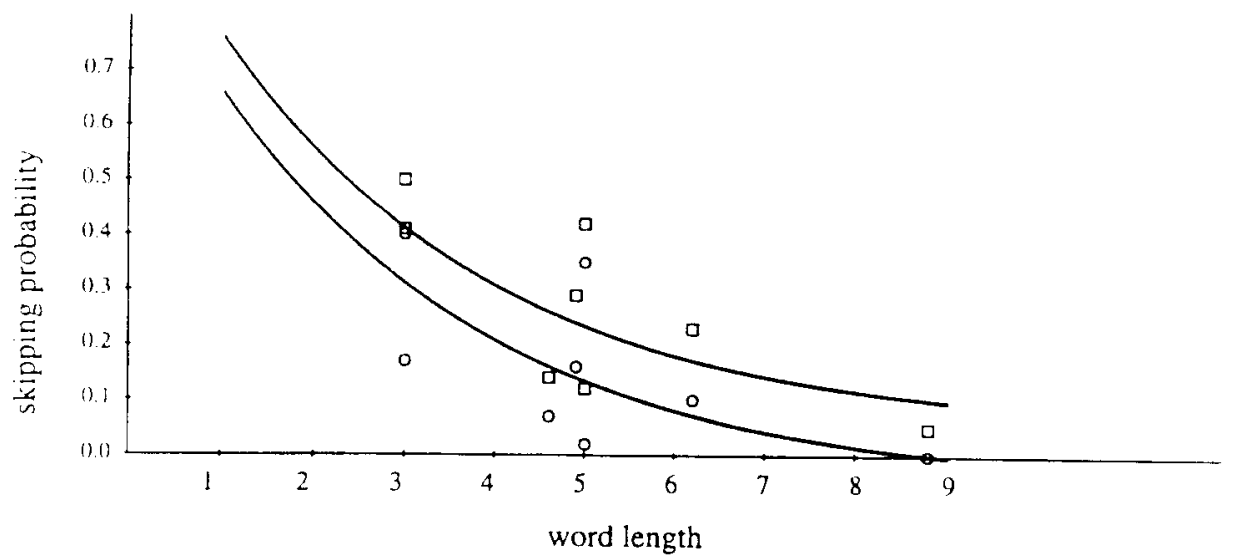

Fig. 4. Skipping rate as a function of word length and contextual constraint (circle = easy

condition; square $=$ difficult condition). Empirical data from Table 2. Fitted curve based on nonlinear regression with exp(word length) and contextual constraint as predictors.

more important predictor of skipping rate than processing load. That is, to predict how often a word was skipped, it is better to know how long the word was than to know whether it was visible in the parafovea, of high frequency, or highly const-rained by the preceding context. Of additional interest is the finding that the regression weight of word length is virtually the same for studies in which word properties were manipulated $(0.36)$ as for studies in which context constraints were manipulated (0.34). So, the empirical evidence strongly points to the conclusion that short words are skipped more often because they are short and not because they are easy. The only thing processing difficulty does, is slightly modulate the basic underlying phenomenon.

\section{Towards a new model of word skipping}

In the previous section we have seen that word skipping is primarily determined by visual factors (such as the length of the upcoming word, and its distance from the launch site; Kerr, 1992). The impact of these factors is not secondary to the probability of recognizing a word with particular language characteristics at a particular distance in the parafovea, although linguistic factors may slightly change the a priori skipping probability. What we need, therefore, is a model of word skipping that is based on visual characteristics of the text.

A problem at this point is that nearly all non-linguistic models of inter-word eye behaviour in reading are characterized by a deterministic scanning strategy. In older 
models, this was a chain of more or less equidistant fixations; in more recent models, it basically is a word-to-word motion. The fact that eye movement patterns in reading rarely exhibit the simple characteristics predicted by these models, has to be explained by the assumption of a (considerable) oculomotor error and occasional intrusions of the linguistic processor. It may be asked, however, whether the assumption of a non-probabilistic oculomotor strategy has not been the major weakness of the oculomotor theories, as it only enables fairly simplistic and coarse predictions about inter-word eye behaviour. At least, it is tempting to see the regular patterns of Figs. 1, 3, and 4 as the result of an underlying stochastic process. According to this view, most words are skipped not because of error in the landing position, but because the system did not "intend" to look at them.

\section{Target selection based on the extended optimal viewing position effect}

To have a stochastic, non-linguistic model of eye movement control in reading, we must be able to predict the probability of word identification as a function of word length and stimulus distance from the fixation location. This is what Brysbaert, Vitu, and Schroyens (1996) investigated. They presented words of different lengths at various positions relative to the fixation location and for different periods of time. Figure 5 depicts their main finding. It shows the probability of recognizing a

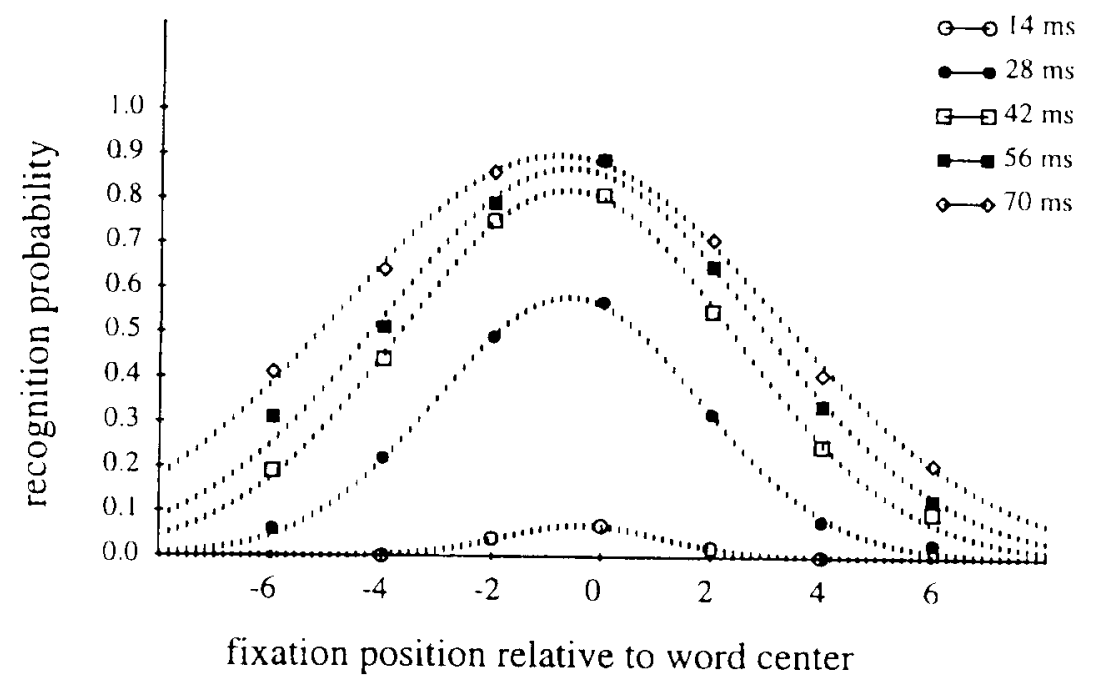

Fig. 5. Probability of word recognition as a function of presentation duration and word position

relative to fixation location. Empirical data and best fitting Gaussian distributions (reprinted from Brysbaert et al., 1996). 
five-letter word on different positions in foveal and parafoveal vision and for time intervals ranging from 14 to $70 \mathrm{~ms}$. For each presentation duration, the probability of recognizing a word at a certain distance could be described reasonably well with a Gaussian distribution that had the mode shifted slightly to the left of the word centre. A similar pattern was found for three-and seven-letter words. Brysbaert et al. (1996) called this the Extended Optimal Viewing Position (EOVP) effect.

Given that the probability of recognizing a word presented at a certain distance and for a given period of time is described reasonably well by a normal curve, this information can be used by the eye guidance system to estimate the chances of identifying the upcoming parafoveal words within the time period of an average fixation (200-220 ms), and to select the most appropriate parafoveal target word. The estimates are based on (1) the length of the word blobs and the distance of the word blobs from the fixation location, and (ii) the standard deviation of the Gaussian EOVP Curve. The latter is a function of text difficulty and task demands (e.g., it will he larger for easy texts and for cursory reading). Although the estimates are rather crude, they give a relatively good idea of what the chances are to recognize an "average word" of a particular length and at a particular distance in the parafovca during an "average fixation duration". It is our contention that this educated guess, which becomes available rather soon in the fixation because it is based on coarse visual information, is used in combination with the ongoing text processing to decide whether or not the next parafoveal word can be skipped. So, a parafoveal word with an estimated probability of $90 \%$ recognition at the end of the current fixation, can most of the time be skipped without implications for the ongoing text processing. Only in a few instances, when processing is hard, is it better not to skip the word. In the long run, this will lead to something like $10 \%$ fixation and $90 \%$ skipping (so that the skipping rate equals the identification probability).

If the above reasoning is correct, then a straightforward prediction follows: We should be able to describe parafoveal target word selection with the use Of a simple inverted Gaussian distribution [i.e., I - exp(-sgr(letterposition-fixation location)/ standard deviation))), as shown in Fig. 6 (note that in this first approach, we disregard the shift of the EOVP curve to the left of foveal word centre). In Fig. 6, we depict the situation for a launch site on the middle of a three-letter word in front of a five-letter parafoveal word. There is a probability of $\mathrm{pI}$ that the foveal threeletter word will be refixated, a probability of $\mathrm{p} 2$-pl that the parafoveal word will he fixated, and a probability of I -p2 that the parafoveal word will be skipped. Note that the function has only one parameter, the magnitude of the standard deviation.

To test the plausibility of our model, we examined the first-pass forward saccades of Brysbaert and Mitchell (1996, Experiment 3; see above) and plotted the landing positions as a function of target word length and launch site. So, for each forward saccade we looked at the length of the words to the right of the launch position (up to 15 letter positions) and noted where the eyes landed relative to those words. The 


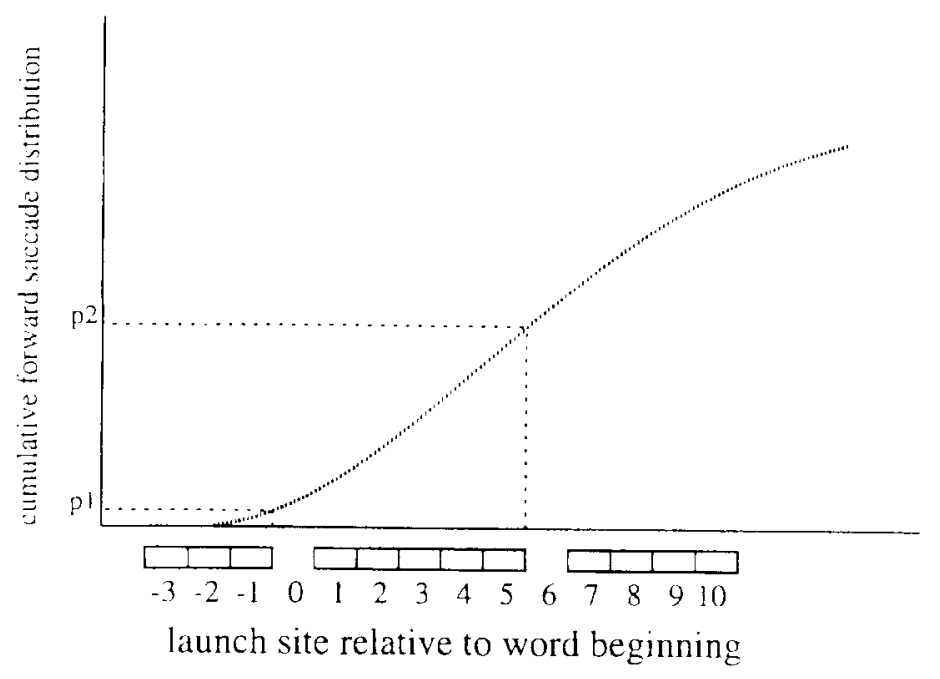

Fig. 6. Graphical display of how an inverted EOVP curve can be used as a cumulative landing position distribution going from the launch site to several words in the right visual half-field.
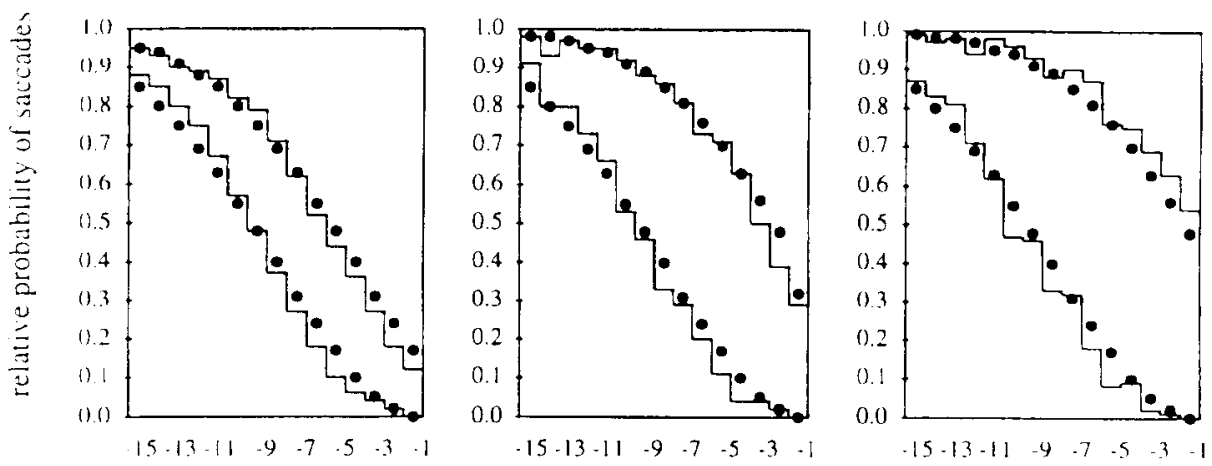

launch site relative to word beginning

Fig. 7. Fit of the mathematical model to empirical data frorn Brysbaert and Mitchell ( 1996,

Experiment 3) as a function of word length and launch site. Left panel: 3-letter target words: middle panel: 5-letter target words; right panel: 7-letter target words. Left down corner of each

panel: probability of landing sites in front of the word; right upper corner of each panel: probability of landing sites behind the word; middle section: probability of landing sites on the word. Lines: empirical values; closed circles: predictions by the model. 
data are plotted in Fig. 7 (horizontal lines) for target words of three, five, and seven letters. So, when a saccade was launched 15 letter positions in front of a three-letter target word, there was $88 \%$ chance that the eyes would land in front of the word, $7 \% \mathrm{c} \%$ chance that they would land on the word, and 5\% chance that they would land behind the word. For a five-letter target word, these probabilities were respectively $91 \% c, 7 \%$, and $2 \%$. For seven-letter words, they were $88 \%, 11 \%$, and I \%, (see Fig. 7, first column of each panel).

We then searched for the best fitting EOVP curve; which happened to have a standard deviation of 10 letter positions (indicated by the black circles in Fig. 7). As can be seen, the empirical data were described remarkably well by a mathematical equation that has but one unknown parameter.

\section{Fleshing out the model}

In the previous paragraph, we have described the general outline of a stochastic non-linguistic eye guidance strategy that can account to a high degree for the observed word skipping rates as a function of word length and launch site. In the present paragraph, we will try to outline some of the assumptions underlying the model.

First, it is important to realize that the probabilities depicted in Fig. 7 do not agree with the distribution of landing positions. They represent the probability that the landing site will be in front of the target word, on the target word, or behind the target word. It is perfectly possible (and quite likely) that the underlying landing site distribution is not normal and unimodal but multimodal with a peak near the middle of each parafoveal word. Otherwise, if the data of Figure 7 represented the landing site distribution, our findings would have been more in line with a model that postulates a constant saccade size and a normally distributed oculornotor error. This is not to say that we reject oculomotor errors as a factor contributing to word skipping (see above). As a matter of fact, simulations indicate that the fit of our model increases significantly if a normally distributed landing error with a standard deviation of two letter positions is added to the model.

Second, it cannot be denied that for the model to work, it is not enough to calculate the identification probabilities of parafoveal words. It is also necessary to "decide" which word to pick out on the basis of these probabilities. At present, it is not clear which processes are involved in the decision and where the decision is made (leaving aside the possibility of a "homonculus problem"). On the one hand, we could think of a version that closely resembles a random control model. According to this version, at the beginning of a fixation, a random number is generated (between 0 and 1) and the word selected that corresponds to this prohability (e.g., if the number falls between $\mathrm{pl}$ and $\mathrm{p} 2$ in Fig. 6, then the parafoveal five-letter word would be selected as target). On the other hand, one could think of a 
version in which the selection depends on the amount of resources left open by the ongoing text processing. As suggested above, we tend to prefer the latter version. This implies that the decision is more likely to be taken by a system related to language processing than by an autonomous oculomotor control system.

Given that we accept a decision based on language processing, it may be asked what the difference still is with prevailing linguistic control theories. According to these theories, a word is skipped because it was recognized during the previous fixation. According to our view, a word is skipped because the language system estimates chances high enough that it will be identified by the end of the current fixation or, at least, that bypassing the word will not hinder text understanding (e.('

because the text fragment is easy). So, the difference is essentially one between an evaluation of what has already been achieved and an educated guess of what will be achieved in the near future. We believe the latter forms the basis of eye guidance in reading, because an educated guess can be realized earlier in the time course of a fixation.

In our view, attentional theories of eye movement control in reading have seriously underestimated the time it takes to identify a parafoveal word, although Rayner himself pointed to the fact that word identification times increase $90 \mathrm{nms}$ per degree of eccentricity (Rayner and Morrison, 1981; see also Schiepers, 1980). This value should be added to the $60 \mathrm{~ms}$ stimulus transfer time from the eyes to the brain and the $100 \mathrm{~ms}$ needed for saccade programming (McConkie, 1983), so that it is virtually impossible for a parafoveal word beginning at an eccentricity of three letter positions to det-ermine the size of the upcoming saccade, unless the fixation lasts considerably longer than $60+100+90=250 \mathrm{~ms}$. What is possible, however, is that the system responsible for the programming of the next saccade at a certain point in time (i.e., before the programming of the saccade starts) estimates the likelihood that the parafoveal word will be recognized within the next $100 \mathrm{~ms}$. In principle, the system could use whatever information is available at that moment (Vita, 1991 h), but most of, the time this will not be very detailed information about the word, but rather some crude measures such as word length and distance in the parafovea (see also below).

At this point, it is necessary to mention explicitly a third assumption of our model, namely that the parafoveal word is processed in parallel with the foveal word, but with a delay (see Schroyens et al., in press, for a more detailed description of our account and some empirical evidence for it). There is little point in estimating the probability that a parafoveal word will be recognized at the end of the current fixation, if processing only starts when the foveal word has been identified (as assumed in current attention theories). Furthermore, if the parafoveal word is processed in parallel with the foveal word, the educated guess can gradually be updated as a function of the duration of the fixation. As shown above, the basic parameters that determine word skipping are word length and launch site. However, the effect of these parameters is to some extent modulated by the difficulty of the 
parafoveal word. One way of conceiving this, is to state that on a few occasions, the (easy) parafoveal word is recognized soon enough before the saccade is launched, so that the programmed saccade to this word can be cancelled and replaced by a saccade to the next word. This seems to be O'Regan's (1990) reasoning, when he claims that linguistic information can guide eye movements if fixation durations are exceptionally Tong. Another view is that the first educated target guess, based on visual factors, is constantly updated by incoming linguistic information about the parafoveal word until saccade programming begins. The advantage of the latter view is that the extra information need not be lexical identification, but can be any sub-lexical information (such as stimulus familiarity, fit of the word blob within the contextual expectations, frequency of the first bigram/trigram, and so on). The main purpose of this extra information is to improve the estimated probability that tile parafoveal word will be recognized at the moment of saccade onset (which takes place some $100 \mathrm{~ms}$ later).

\section{Some nevi, predictions}

There are three main predictions to be made on the basis of our model of word skipping. The first is that differences in inter-word eye behaviour as a function of text difficulty and expertise can be described by a change in the standard deviation of the Extended Optimal Viewing Position curve. This would mean that exactly the same mechanisms are used in different reading situations and that differences in eye behaviour are gradual rather than absolute (the latter is the case, for instance, in the distinction between a word-by-word strategy and a skip-every-second-word strategy).

The second prediction is that because word skipping happens on the basis of partial information, in a non-neglectable number of instances words will be skipped erroneously. A word that on the basis of its length and distance looked like a safe bet to skip, may turn out to be more difficult and crucial for the understanding of the text than expected at first sight. In those cases, the skipping is likely to be followed by a regressive eye movement. Indeed, as documented in Chapter 5, the pattern of words initially skipped and immediately afterwards regressed to, occurs very regularly in reading and is probably too frequent to be ascribed to oculomotor error alone. In addition, many of these regressions are preceded by short fixations. For instance, in our eye movement data base of Brysbaert and Mitchell (see above), 35\% of the fixation durations shorter than $200 \mathrm{~ms}$ were followed by a regression to the previous (skipped) word. This was more than the percentage of forward movements to the next word $(23 \%)$ or to the second next word $(27 \%)$. On the other hand, fixations of $300 \mathrm{~ms}$ and more had a chance of less than $15 \%$ to be followed by a regression to the previous word, and more than $30 \%$ to be succeeded by a forward movement to the next word or to the second next word. This finding clearly challenges the predictions 
made by the attention theory (e.g., Rayner, 1995) that short fixations should he followed by a forward movement (because they are a sign that the parafoveal word was identified before the saccade started but after the execution deadline expired). The finding also presents problems for the "first-fixation-duration" measure often used in eye movement research, as the duration of a fixation on a target word followed by a regression to the previous skipped word, is unlikely to reveal much about the processing of the target word.

The data on regressive eye movements after short fixation durations bring us to the third prediction of the model, namely that language influences on word skipping may be due to a shortening of an intended saccade rather than to a lengthening. According to most current models of eye movement control in reading, the initial target for a forward saccade is the next parafoveal word. So, any linguistic influence must be limited to a replacement of this saccade by a saccade to a more distant word. However, if the target of the initial saccade can be the second or even the third word in the parafovea, as our model claims, then linguistic influences can result in a cancelling of an intended skip and its replacement by a saccade to a less distant word. This would happen, for instance, when the eye guidance system originally estimated that a particular parafoveal word could safely be skipped, but in the time course of the fixation noticed that this was unlikely to be so (e.g., because the processing rate of the parafoveal word was slower than anticipated). Cancelling an intended skip may even be a more common language processing intervention than replacing a saccade by a saccade to a more distant word, as there is some evidence that shortening a saccade is easier than lengthening it (Becker, 1991, pp. 129-I 30).

\section{Conclusion}

It is tempting to think of eye movements during reading as an activity which is completely regulated by either a dumb oculomotor strategy or by the ongoing text processing. In both cases, the pattern of fixations within a line of text seems needlessly complicated and chaotic, and requires the assumption of a large landing position error.

Another approach is to see eye guidance as hypothesis generation on the basis of incomplete information. In this view, errors (and, hence, corrective movements) are an inherent part of the model and should exhibit properties related to the hypothesis generator. We have tried to show that at least word skipping can he described quite well within such a framework. 


\section{Acknowledgements}

The collaboration leading to this text was made possible by grants from the Fonds voor Wetenschappelijk Onderzoek and the European Union (BIOMED BMIII-CT94-1441) to the first author, a grant from the Fyssen Fondation to the second author, and a Tournesol grant (T/94.046) fostering collaboration between France and Flanders. We would like to thank Keith Rayner, Ralph Radach, and an anon-ymous reviewer for helpful comments on an earlier draft.

\section{References}

Balota, D.A., Pollatsek, A. and Rayner, K. (1985). The interaction of contextual constraints and parafoveal visual information in reading. Cognitive Psychology, 17, 364-390. Becker, W. (1991). Saccades. In: R.H.S. Carpenter (Ed.), Vision and Visual Dysfunction.

Vol. 8: Eye Movements. London: MacMillan, pp. 95-137)

Blanchard, H.E., Pollatsek, A. and Rayner, K. (1989). The acquisition of parafovcal word information in reading. Perception and Psychophysics, 46, 85-94.

Bouma, H. and De Voogd, A.H. (1974). On the control of eye saccades in reading. Vision Research, 14, 273-284.

Bryshaert, M. and Mitchell, D.C. (1996). Modifier attachment in sentence parsing: Evidence from Dutch. The Quarterly Journal of Experimental Psychology, 49A, 664-695.

Bryshaert, M. and Vitu, F. (1995). Word skipping: Its implications for theories of eye move-ments in reading. Paper presented at the Eighth European Conference on Eye Move-ments. Derby, UK.

Brysbaert, M., Vitu, F. and Schroyens, W. (1996). The right visual field advantage and the optimal viewing position effect: On the relation between fovea) and parafovcal word recognition. Neuropsychology, 10, 385-395.

Buswell (1920) An experimental study of eye-voice span in reading. Supplementary Educa-tional Monographs, 17.

Coeffe. C. and O'Regan, J.K. (1987). Reducing the influence of nontarget stimuli on saccade accuracy: Predictability and latency effects. Vision Research, 27, 227-240.

Ehrlich, S.F. and Rayner, K. (1981). Contextual effects on word perception and eye movements during reading. Journal of Verbal Learning and Verbal Behavior, 20, 641-655. lardmann, B.

and Dodge, R. (1898). Psychologische Untersuchungen uber das I.esen ${ }^{\text {al-If }} \mathrm{cx}-$ perimenteller Grundlage. Halle.

Findlay, J.M. (1982). Global visual processing for saccadic eye movements. Vision Re-search, 22, 10331045.

Henderson, J.M. and Ferreira, F. (1990). Effects of fovea) processing difficulty on the per-ceptual span in reading: Implications for attention and eye movement control. .Journal of Experimental Psychology: Learning, Memory, and Cognition, 16, 417-429.

Henderson, J.M and Ferreira, F. (1993). Eye movement control during reading: Fixation measures reflect fovea) but not parafoveal processing difficulty. Canadian Journal of Experimental Psychology, 47, 201-221. 
Hochberg, J. (1975). On the control of eye saccades in reading. Vision Research, 15, 620. Hochberg, J.

(1976). Toward a speech-plan eye-movement model of reading. In R.A. Monty and J.W. Senders (Eds.), Eye movements and psychological processes. Hillsdale, NJ: Erlbaum.

Huey, E.B. (1908). The psychology and pedagogy of reading. New York: MacMillan. Hyona,.1.( 1993).

Effects of thematic and lexical priming on readers' eye movements. Scandinavian Journal of Psychology, 34, 293-304.

Inhoff, A.W. and Topolski, R. (1994). Use of phonological codes during eye fixations in reading and in on-line and delayed naming tasks. Journal of Memory and Language. 33, 689-713.

.lust, M.A. and Carpenter, P.A. (1980). A theory of reading: From eye fixations to comprehension. Psychological Review, 87, 329-354.

.lust, M.A. and Carpenter, P.A. (1987). The psychology of reading and language comprehension. Newtown, MA: Allyn and Bacon.

Kerr, P.W. (1992). Eye movement control during reading: The selection of where to send the eyes. Doctoral Dissertation, University of Illinois at Urbana-Champaign, Urbana, IL.

McConkie, G.W. (1979). On the role and control of eye movements in reading. In: P.A. Kol-ers, M.E. Wrolstad and H. Bouma (Eds.), Processing of Visible Language (VoI. I , pp. 37-48). New York: Plenum.

McConkie, G.W. (1983). Eye movements and perception during reading. In: K. Rayner (Ed.), Eye Movements in Reading: Perceptual and Language Processed. New York: Academic Press, pp. 65-96.

McConkie, G.W., Kerr, P.W., Reddix, M.D. and Zola, D. (1988). Eye movement control during reading: I. The location of initial eye fixations. Vision Research, 28, 1107-1 118.

McConkie, G.W., Kerr, P.W. and Dyre, B.P. (1994). What are 'normal' eye movements dur-ing reading: Toward a mathematical description. In: J. Ygge and G. I ennerstrand (Eds.), Eye Movements in Reading. Oxford: Elsevier, pp. 315-327.

Morrison, R.E. (1984). Manipulation of stimulus onset delay in reading: Evidence for paral-lel programming of saccades. Journal of Experimental Psychology: Human Perception and Performance, $10,667-682$

O' Regan, J.K. (1979). Eye guidance in reading: Evidence for the linguistic control hypothe-sis. Perception and Psychophysics, 25, 501-509.

O' Regan, J.K. (1980). The control of saccade size and fixation duration in reading: The lim-its of' linguistic control. Perception and Psychophysics, 28, 112-1 17.

O' Regan, J.K. (1990). Eye movements and reading. In: E. Kowler (Ed.), Eye Movements and Their Role in Visual and Cognitive Processes. Amsterdam: Elsevier, pp. 395153.

Pollatsek, A., Lesch, M., Morris, R.K. and Rayner, K. (1992). Phonological codes are used in the integration of information across saccades in word identification and reading. Journal of Experimental Psychology: Human Perception and Performance, 18, 148- 162.

Pollatsek, A. and Rayner, K. (1990). Eye movements and lexical access in reading. In: D.A. Balota, G.B. Flores d'Arcais and K. Rayner (Eds.), Comprehension Processes in Read-ing. Hillsdale, NJ: Erlbaum, pp. 143-163.

Radach, R. and Kempe, V. (1993). An individual analysis of initial fixation positions in reading. In: G. d'Ydewalle and J. Van Rensbergen (Eds.), Perception and Cognition: Advances in Eye Research. Amsterdam: North-Holland, pp. 213-226. 
Rayner, K. (1978). Eye movements in reading and information processing. Psychological Bulletin, 85, 618-660.

Rayner, K. (1995). Eye movements and cognitive processes in reading, visual search, and scene perception. In: J.M. Find Iay, R.W. Kentridge and R. Walker (Eds.), Eye Movement Research: Mechanisms, Processes, and Applications. Amsterdam: North-Holland, pp-3-22.

Rayner, K. and Fischer, M.H. (1996). Mindless reading revisited: Eye movements during reading and scanning are different. Perception and Psychophysics, 58. 734-747.

Rayner, K. and Morrison, R.E. (1981). Eye movements and identifying words in paratovcal vision. Bulletin of the Psychonomic Society, 17, 135-138.

Rayner, K. and Raney, G.E. (1996). Eye movement control in reading and visual search: Ef-fects of word frequency. Psychonomic Bulletin and Review, 3. 245-248.

Ravner, K., Sereno, S.C. and Raney, G.E. (1996). Eye movement control in reading: A com-parison of two types of models. Journal of Experimental Psychology: Human Percep-tion and Performance, 22, $1188-1200$

Rayner, K. and Well, A.D. (1996). Effects of contextual constraint on eye movements in reading: A further examination. Psychonomic Bulletin and Review, 3, 504-509.

Schiepers, C. (1980). Response latency and accuracy in visual word recognition. Perception and Psychophysics, 27, 71-81.

Schroycns, W., Vitu, F., Brysbaert, M. and d'Ydewalle, G. (in press). Visual attention and eye-movement control during reading: The case of parafoveal processing. '['he Quar-terly Journal of Experimental Psychology.

Schustack, M., Ehrlich, S. F. and Rayner, K (1987). The complexity of contextual facilita-tion in reading: Local and global influences. Journal of Memory and Language, 26, 322-340.

Schwanenflugel, P.J. (1986). Completion norms for final words of sentences using a multi-pie production measure. Behavior Research Methods, Instruments and Computers, 18, 363--371.

Shchilske, W. (1975) Reading eye movements from an information-processing point of view. In: D. Massaro (Ed.), Understanding Language. New York: Academic Press, pp. 291-311.

Vitu, F. (1991 a). The existence of a center of gravity effect during reading. Vision Research, 31,12891313.

Vitu, F. (1991 b). The influence of parafoveal preprocessing and linguistic context on the op-timal landing position effect. Perception and Psychophysics, 50, 58-75.

Vitu, F., O'Regan, J.K., Inhoff, A.W. and Topolski, R. (1995). Mindless reading: Eye move-ment characteristics are similar in scanning letter strings and reading texts. Perception and Psychophysics, $57,352-364$

Vonk, W. (1984). Eye movements during comprehension of pronouns. In: A.G. Gale and F. Johnson (Eds.), Theoretical and Applied Aspects of Eye Movement Research. Amster-dam: North-Holland. 